\title{
Personalização de informação para uso de medicamentos para idosos autônomos polimedicados
}

\author{
Drug use information personalization for autonomous elderly polymedicated
}

SILVA, Cláudio Henrique da; Doutor; UFPR

design@midiak.com.br

SPINILLO, Carla Galvão; Doutora; UFPR

cgspinillo@gmail.com

\section{Resumo}

O envelhecimento da população brasileira é cada vez maior e traz consigo um maior número de pessoas convivendo com doenças crônicas, muitos das quais fazendo uso de vários medicamentos, isto é, polimedicados. $\mathrm{O}$ uso inadequado dos medicamentos pode trazer sérias consequências para idosos polimedicados e a personalização das informações pode melhorar significativamente a adesão ao seu tratamento. Neste sentido, o objetivo deste artigo é examinar a personalização de informação de uso de medicamentos para idosos polimedicados destacando a presença recorrente de informações visuais neste processo. Três soluções de personalização de informação de uso de medicamentos, especificamente desenvolvidas para idosos polimedicados, são apresentadas e discutidas. A partir desta discussão, algumas questões como a importância da participação do idoso no processo personalização e a relevância de informações visuais na personalização são apresentadas como possíveis desmembramentos.

Palavras Chave: personalização da informação em saúde; idoso; polimedicação e adesão ao medicamento.

\begin{abstract}
The aging of the population is increasing and brings with it a greater number of people living with chronic diseases, many of which making use of several drugs, i.e. polymedicated. The drug misuse can have serious consequences for polymedicated elderly and information personalization can significantly improve adherence to treatment. In this sense, the objective of this article is to examine the drug use information personalization for polymedicated elderly highlighting the recurring presence of visual information in the process. Three solutions of drug use information personalization, specifically designed for polymedicated elderly, are presented and discussed. From this discussion, some issues such as the importance of the elderly participation in the personalization process and the relevance of visual information in the personalization are presented as possible dismemberment.
\end{abstract}

Keywords: health information personalization; elderly; polymedication and medication adherence. 


\section{Introdução}

A população brasileira começa a acompanhar uma tendência mundial no que diz respeito ao envelhecimento. Isto é o que mostram os dados do último Censo Demográfico 2010 realizado pelo Instituto Brasileiro de Geografia e Estatística - IBGE (PORTAL BRASIL, 2014 onde se observa um aumento no número de idosos com idade acima de 65 anos e diminuição do contingente de pessoas com até 20 anos. Em 2010, o percentual de idosos subiu para 7,4\% da população brasileira, contra 5,9\% em 2000 e 4,8\% em 1991. Segundo Simões (2012, p. 111) "em 2008, para cada grupo de 100 crianças de 0 a 14 anos existiam 24,7 idosos de 65 anos ou mais. Em 2050, o quadro mudará drasticamente e para cada 100 crianças de 0 a 14 anos existirão 172,7 idosos."

Este cenário evidencia um grande desafio para os idosos no mundo e em particular no Brasil: envelhecer com qualidade de vida. Embora a expectativa de vida do brasileiro tenha aumentado (IBGE, 2014, o envelhecimento traz consigo a convivência com doenças crônicas, herdadas geneticamente ou adquiridas pelo estilo de vida de cada pessoa. Cazarim e Araújo (2011, p. 306) comentam que mais de $80 \%$ da população idosa toma, no mínimo, um medicamento diariamente em virtude de alguma doença crônica. Destes $80 \%$, estima-se que $20 \%$ seja polimedicado (SIMÕES, 2012). Como consequência, a qualidade de vida de idosos com doenças crônicas depende de tomar de forma adequada os seus múltiplos medicamentos, isto é, de sua adesão ao tratamento prescrito pelo médico. Contudo, a não-adesão ao tratamento entre os idosos é um problema comum (GELLAD ET AL., 2011), principalmente quando são polimedicados (OLESEN ET AL., 2013). As consequências da não-adesão ao tratamento podem ser graves, chegando a internações e até óbito do paciente.

Se por um lado a adesão ao tratamento é vista como importante para a qualidade de vida de idosos polimedicados na medida em que lidam melhor com suas doenças crônicas, a personalização da informação de uso de medicamentos pode ser uma forma de auxilia-los a tomar corretamente os medicamentos prescritos.

Diante do exposto, este artigo discute a personalização de informação de uso de medicamentos para idosos polimedicados, destacando a presença recorrente de informações visuais neste processo. Para isto, são abordados os temas de adesão ao medicamento, polimedicação como um dos fatores de não-adesão e personalização da informação. Em seguida são apresentados três exemplos de personalização de informação para uso de medicamentos destinados a idosos polimedicados. Por fim, considerações e questões são propostas como fruto da reflexão sobre a personalização de informação de uso de medicamentos por idosos polimedicados.

\section{Adesão ao medicamento}

De acordo com a Organização Mundial de Saúde - OMS (WHO, 2003, p.3), a adesão é observada "na medida em que o comportamento de uma pessoa - ao tomar medicação, seguindo uma dieta, e/ou a mudança de seu estilo de vida - coincide com as recomendações de um profissional de saúde" 2003, p. 3) Ela apresenta a adesão como um fenômeno dimensional, com 5 fatores ou dimensões (Figura 1): relacionados ao paciente, socioeconômicos, profissionais e sistema de saúde, condições (em relação à doença) e terapia. Embora os fatores relacionados ao paciente sejam considerados determinantes, atribuir somente ao paciente a responsabilidade pelo seu tratamento e por tomar os seus medicamentos é uma grande fonte de confusão, uma vez que cada fator ou dimensão também influencia no processo (WHO, 2003). 
Figura 1 - As 5 dimensões da adesão

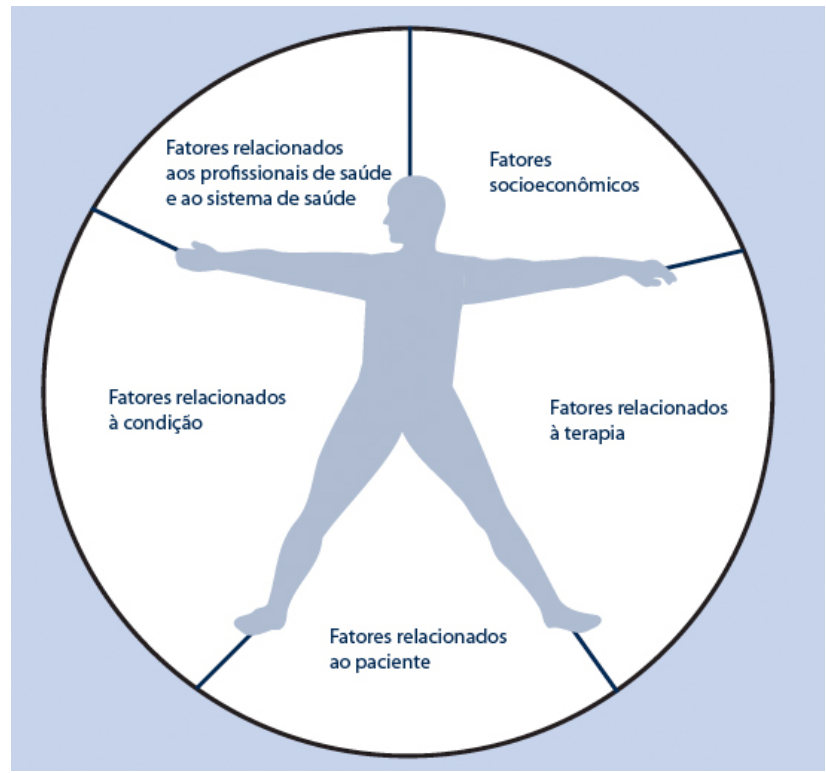

Fonte: Traduzido de WHO (2003, p.27)

Fatores socioeconômicos: em países ou locais com baixo nível de desenvolvimento socioeconômico o paciente pode ser colocado em posição de ter de escolher entre prioridades concorrentes. Tais prioridades incluem frequentemente exigências para direcionar os recursos limitados disponíveis para atender às necessidades de outros membros da família, como as crianças ou os pais, com os quais eles se preocupam.

Fatores relacionados aos profissionais de saúde e ao sistema de saúde: refere-se a relação paciente-profissionais de saúde e também o sistema de saúde. Fatores que influenciam negativamente incluem serviços de saúde pouco desenvolvidos, com reembolso inadequado ou inexistente pelos planos de saúde, sistemas de distribuição de medicamentos ruins, a falta de conhecimento e treinamento para profissionais de saúde sobre o gerenciamento de doenças crônicas, prestadores de cuidados de saúde sobrecarregados de trabalho, a falta de incentivos e feedback sobre o desempenho, consultas de curta duração, a fraca capacidade do sistema para educar os pacientes e fornecer acompanhamento, incapacidade de estabelecer o apoio da comunidade e capacidade de auto-gestão, falta de conhecimento sobre a adesão e de intervenções eficazes para melhorá-lo.

Fatores relacionados à condição: são aqueles que representam demandas particulares relacionados com a doença enfrentada pelo paciente. Os que são fortes determinantes da adesão são aqueles relacionados com a gravidade dos sintomas, o nível de deficiência (física, psicológica, social e profissional), a taxa de progressão e gravidade da doença, e da disponibilidade de tratamentos eficazes. O seu impacto depende de como eles influenciam a percepção dos pacientes de risco, a importância de seguir o tratamento, bem como a prioridade colocada sobre a adesão.

Fatores relacionados à terapia: destacam-se a complexidade do regime médico, a duração do tratamento, as falhas de tratamento anteriores, mudanças freqüentes do tratamento, 0 imediatismo de efeitos benéficos, os efeitos colaterais e a disponibilidade de apoio médico para lidar com eles. 
Fatores relacionados ao paciente: representam os recursos, conhecimentos, atitudes, crenças, percepções e expectativas do paciente. Os mais comuns são: esquecimento; estresse psicossocial; ansiedades sobre possíveis efeitos adversos; baixa motivação; conhecimento e habilidade inadequados em gerenciar os sintomas e tratamento de doenças; falta de autopercepção da necessidade de tratamento; falta de efeito percebido de tratamento; crenças negativas a respeito da eficácia do tratamento; incompreensão e não aceitação da doença; descrença no diagnóstico; falta de percepção do risco para a saúde relacionados com a doença; má compreensão das instruções de tratamento; falta de aceitação de uma monitoração; baixas expectativas de tratamento; desesperança e sentimentos negativos; frustração com prestadores de cuidados de saúde; medo de dependência; ansiedade sobre a complexidade do regime de medicamentos e sentimento estigmatizado pela doença.

Em relação aos idosos polimedicados, Werlang, Argimon e Stein (2008) explicam que a nãoadesão à prescrição médica pode ser intencional (quando o paciente deliberadamente não cumpre o seu regime terapêutico) ou não-intencional (devido a limitações físicas, aspectos financeiros e cognitivos), sendo esta a maior causa. Não-adesão ao medicamento é um problema comum entre os idosos (GELLAD ET AL., 2011). Schimtt Jr et al. (2013, p. 616) concordam dizendo que "o desafio da adesão terapêutica torna-se maior quando o paciente é idoso. Esta parcela da população chega a constituir $50 \%$ dos multiusuários de medicamentos devido ao aumento da prevalência de doenças crônico-degenerativas." Olesen et al. (2013) afirmam que idosos polimedicados são mais propensos a não aderirem ao tratamento. Entre as causas figuram reações adversas, polimedicação, baixo conhecimento em saúde e funções cognitivas como esquecimento e falta de atenção. Esquecimento é identificado como a principal causa de não-aderência entre idosos (UNNI; FARRIS, 2011) e potencializada pela polimedicação.

A polimedicação (ou polifarmácia) é uma condição que dificulta significativamente a adesão do paciente ao seu tratamento. Schimtt Júnior et al. (2013, p. 619) dizem que "há de se considerar que um maior número de medicamentos prescritos aumenta significativamente a complexidade do regime terapêutico, ambos aumentando com o avançar da idade." A interação medicamentosa é um fator que contribui para o aumento dessa complexidade e, associada à polimedicação e mudanças fisiológicas no organismo advindas no processo de envelhecimento, faz com que se deva ter maior cuidado na utilização de medicamentos por idosos (SIMÕES, 2012). Sintomas indesejados podem ocorrer como resultado destes fatores, pois os medicamentos podem exercer alguma interferência um no outro, agravado pela mudança do metabolismo do paciente idoso. Relacionando as reações adversas com a não-adesão ao medicamento, Schimtt Júnior et al. (2013, p. 619) comentam que quanto mais medicamentos são utilizados maior é a probabilidade do paciente ter reações adversas e interromper o tratamento.

A adesão ao tratamento terapêutico é um grande desafio para os profissionais de saúde principalmente em relação a pacientes idosos polimedicados, uma vez que para estes pacientes inúmeros fatores fazem com que a adesão tenha a tendência de ser significativamente menor. Neste contexto, a personalização de informação para uso de medicamentos se apresenta como uma forte aliada.

\section{Personalização de informação para uso de medicamentos}

A Organização Mundial de Saúde preconiza que

13 Congresso Brasileiro de Pesquisa e Desenvolvimento em Design, Univille, Joinville (SC) 
os pacientes precisam ser informados, motivados e qualificados para a utilização de estratégias cognitivas e comportamentais de auto-regulação para que possam lidar eficazmente com as demandas relacionadas ao tratamento impostas por sua doença. Para a prestação eficaz de tratamento das condições crônicas, é necessário ativar o paciente e a comunidade que apoia (WHO, 2003, p. 35).

Neste contexto, ativar o paciente significa envolve-lo efetivamente no seu tratamento, o que faz da personalização um aspecto crucial neste processo. Tratar de personalização, no contexto de saúde, é uma tarefa árdua seja pela amplitude do tema, seja pelas características intrínsecas ao processo de personalização.

Como dito, a personalização relacionada à saúde é um tema bastante amplo. A literatura apresenta uma diversidade de termos, cada qual com o seu alcance e especificidade. Personalização do Sistema de Saúde (Personalization of Health Systems), que significa que toda a estrutura do sistema de saúde é revista e remodelada permitindo a personalização da saúde em todos os níveis e serviços (SNOWDON; SCHNARR; ALESSI, 2014). Medicina Personalizada (Personalized Medicine) diz respeito à genética visando personalizar os medicamentos (MINVIELLE ET AL., 2014). Saúde Centrada no Paciente (Patient-Centered Care - PCC), onde o foco reside no paciente, suas demandas e necessidades, e não em sua patologia (MINVIELLE ET AL., 2014; SPATZ, 2009). Informações Pessoais de Saúde (Personal Information Health - PIH); personalização da informação de saúde (tailor health information) e informação personalizada de saúde (personalized health information). Esta última faz parte do modelo de Saúde Centrada no Paciente (SPATZ, 2009; ENWALD ET AL., 2011).

Embora uma sistematização dos termos, identificando seu alcance e suas relações, possa trazer muitas contribuições ao tema, esta tarefa encontra-se fora do escopo deste artigo e não será aprofundada. Partindo-se do questionamento sobre o que seria importante para idosos polimedicados em relação à personalização de saúde, optou-se por examinar a personalização de informação, no contexto de informação personalizada de saúde, mas especificamente sobre uso de medicamentos. Como foi abordado anteriormente, uma grande dificuldade encontrada por idosos polimedicados diz respeito a conseguir tomar adequadamente seus medicamentos diários.

Neste sentido, é importante destacar que não se está tratando de informação de saúde em geral, mas de um momento específico que se refere ao uso do medicamento ou da tarefa de tomar o medicamento. Guenette e Moisan (2011) comentam que os idosos demonstram ter bom conhecimento sobre os medicamentos que tomam, sejam eles prescritos ou não. No entanto, este conhecimento deve envolver também o uso dos medicamentos. As dificuldades enfrentadas pelos idosos polimedicados para tomar seus medicamentos conduzem à ideia de que as informações endereçadas a este público sejam adequadas às suas características, considerando suas limitações físicas, cognitivas, culturais e outras que possam de alguma forma influenciar a leitura e compreensão de tais informações (MCLAUGHLIN; MAYHORN, 2014; CAVACO; SANTOS, 2012; LIU; KEMPER; MCDOWD, 2009). Vários desafios se apresentam diante da ideia de personalizar informações sobre medicamentos.

O primeiro desafio refere-se à padronização dos tratamentos. Na área de Saúde, especificamente, busca-se trabalhar voltado a evidências, isto é, a partir de resultados experimentais. Desta forma, os tratamentos para doenças crônicas são padronizados a partir das evidências encontradas nos estudos. Minvielle et al. (2014) comentam que existe uma padronização (standardization) de tratamentos e orientações para doenças crônicas em idosos. 
No entanto, a adesão aos medicamentos depende também de uma personalização ou individualização da estratégia de tomada de medicamentos. Cada idoso encontra-se em um contexto específico (cultural, comportamental, social, econômico), além de estar em um regime terapêutico adequado às suas condições. Bosworth et al. (2011) acreditam que intervenções mais eficazes fazem uso de uma combinação de abordagens para lidar com os diversos tipos de barreiras à adesão aos medicamentos. Considerando-se que cada idoso polimedicado possui um regime terapêutico específico e um contexto próprio de uso de medicamentos é possível afirmar que tanto os materiais quanto as abordagens para melhorar sua adesão ao medicamento precisam ser individualizados ou personalizados. É recomendável a combinação de um conjunto individualizado de intervenções educacionais e comportamentais para atender às necessidades de um paciente específico para a adesão (MALONEY, KAGAN, 2011). Bosworth et al. (2011) afirmam que além de saberem o que fazer e por quê fazer, os pacientes precisam de habilidades comportamentais para facilitar a adoção e incorporação da tarefa de tomada de medicamentos na sua vida cotidiana. Minvielle et al. (2014) ainda lembram que do ponto de vista do paciente a personalização do cuidado sempre foi um aspecto importante da qualidade na área da saúde. De acordo com os autores, todo paciente quer sentir que está recebendo os cuidados adaptados às suas necessidades particulares. Neste sentido, Pfaff et al. (2010) apontam a padronização como uma barreira à autonomia e ao comportamento proativo do idoso.

Um segundo desafio refere-se a conciliar interesses dos diversos stakeholders envolvidos no uso do medicamento. Bosworth et al. (2011) apontam como passo inicial buscar um entendimento comum de questões-chave dos stakeholders envolvidos, tais como órgãos de governo, indústrias (farmacêuticas, de embalagens), operadoras e serviços de saúde, empregadores, profissionais de saúde e os próprios pacientes.

Deste segundo desafio, surge um terceiro que envolve conciliar regulamentação e custos, além das necessidades de informação individuais de cada stakeholder.

Por fim, Minvielle et al. (2014) comentam que não há uma abordagem integradora global para personalização na área da saúde. Os autores comentam que várias iniciativas têm sido feitas, mas não que não dialogam entre si.

\section{Exemplos de personalização}

Foram selecionados três exemplos de personalização: [1] Clear Rx Medication System, desenvolvido por designer Deborah Adler (ADLER DESIGN, 2014), [2] SUPERMED, desenvolvido pela enfermeira Liliana Batista Vieira (VIEIRA, 2013) e [3] uma solução desenvolvida por uma idosa ${ }^{1}$.

\subsection{Clear Rx Medication System}

Adler desenvolveu um sistema chamado Clear Rx Medication (ADLER DESIGN, 2018) a partir de um incidente onde sua avó tomou por engano o medicamento do seu avô. Este sistema conta com anéis coloridos para diferenciar os membros da família, rótulos intuitivos e de fácil leitura (Figura 2).

\footnotetext{
${ }^{1}$ Esta solução foi observada durante entrevista informal, como parte da pesquisa de doutoramento do autor.
} 


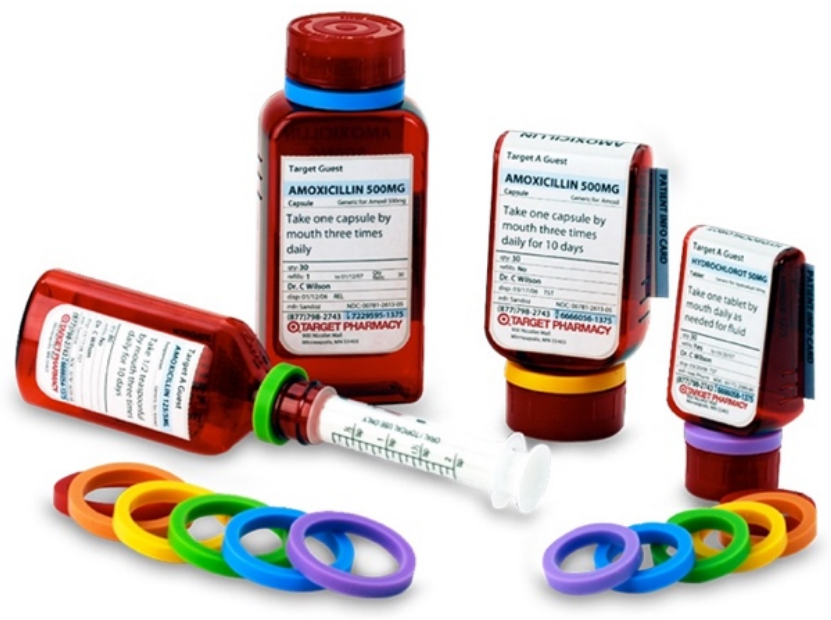

Fonte: Adler Design (2018)

A figura 3 apresenta os elementos adotados neste projeto (BERNARD, 2018: (1) o nome do medicamento é impresso no topo, bem visível; (2) uso da cor vermelha (indicando cuidado) na embalagem; (3) hierarquia da informação, priorizando informações sobre dosagem e como tomar; (4) embalagem de cabeça para baixo, gerando economia de papel pela forma como é aplicado na embalagem; (5) Anel colorido para diferenciar membros da família; (6) cartão para o paciente, com informações detalhadas sobre o medicamento; (7) Adler utilizou a palavra daily (diariamente) ao invés de once (uma vez) para evitar confusões na língua espanhola, onde once significa "onze"; (8) Avisos claros, com a utilização de um conjunto de signos que ela selecionou e refez, juntamente com outro designer (Figura 4).

Figura 3: Elementos do sistema.
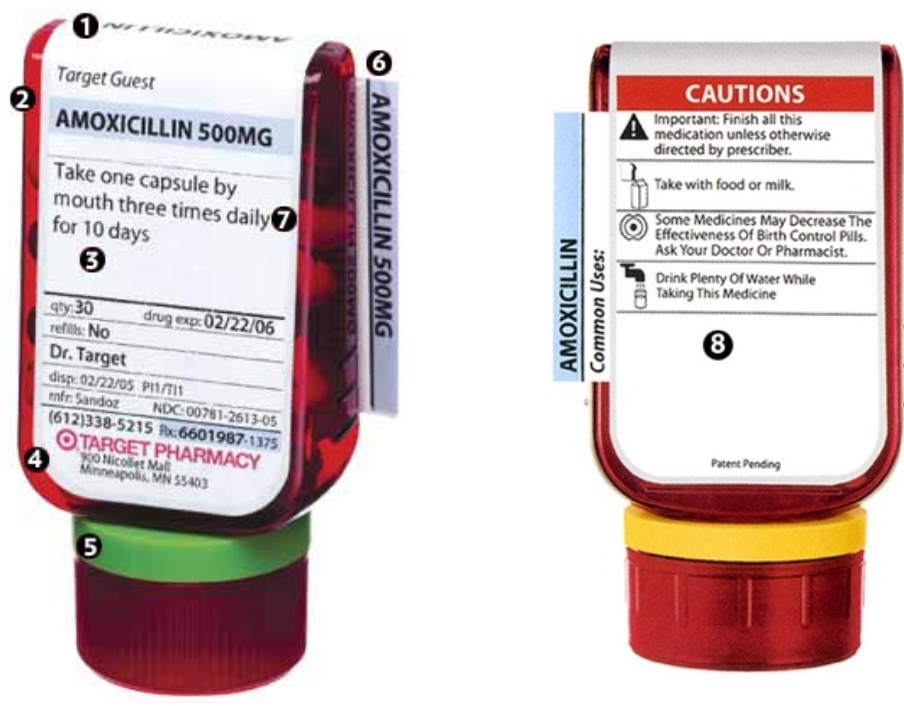

Fonte: Bernard (2018) 
Figura 4: Conjunto de símbolos.

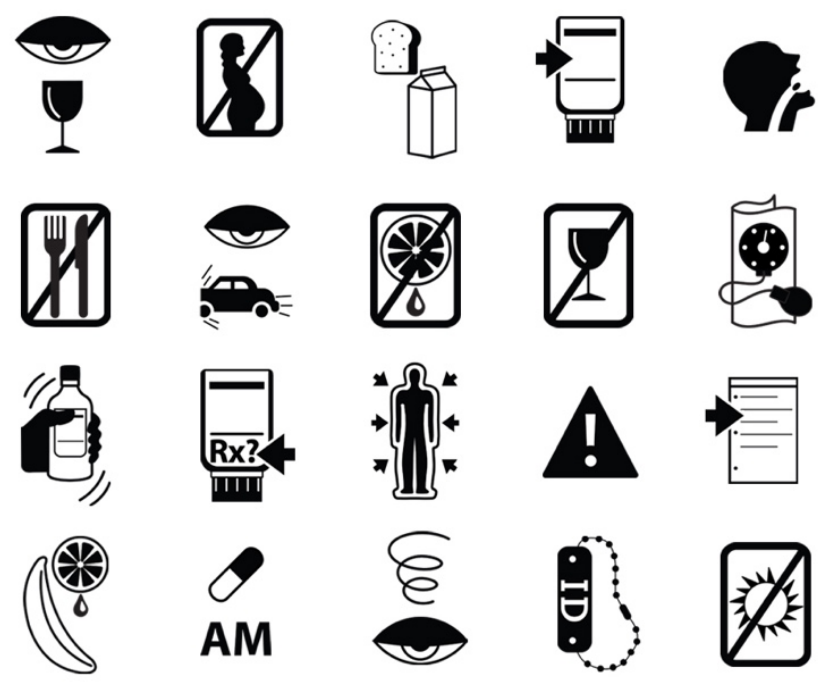

Fonte: Adler Design (2018)

Considerando que pacientes idosos podem ter déficits visuais, Adler acrescentou ao projeto um cartão plástico que funciona como uma lupa, permitindo ampliar as informações do rótulo do medicamento (Figura 5).

Figura 5: Lupa para leitura.
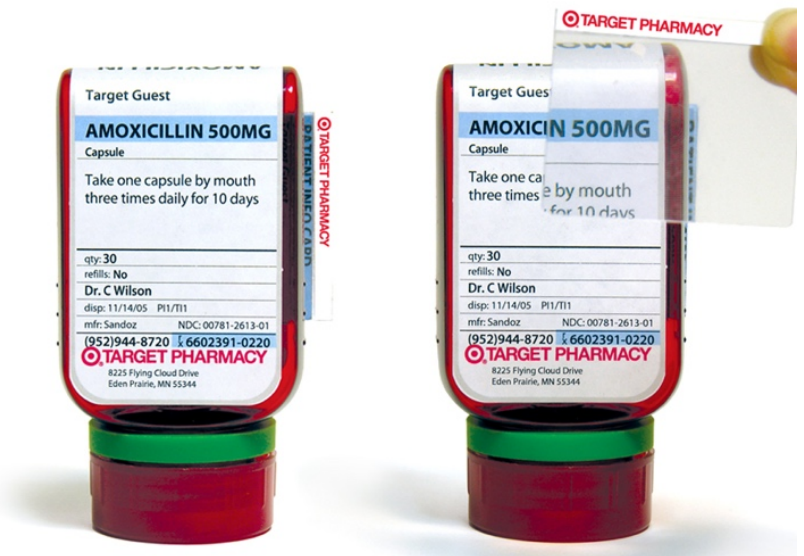

Fonte: Adler Design (2014)

\subsection{Sistema Eletrônico de Uso Personalizado e Controlado de Medicamentos (SUPERMED)}

O Sistema Eletrônico de Uso Personalizado e Controlado de Medicamentos (SUPERMED) foi desenvolvido como parte da tese de Liliana Batista Vieira apresentada à Escola de Enfermagem de Ribeirão Preto da Universidade de São Paulo (USP). Segundo a autora, o seu objetivo é "contribuir 
com a adesão ao tratamento medicamentoso e com a segurança dos pacientes idosos." (VIEIRA, 2013, p. 44) Trata-se de um organizador de medicamentos com alarme para lembrar o paciente sobre o horário correto (Figura 6).

Figura 6: SUPERMED

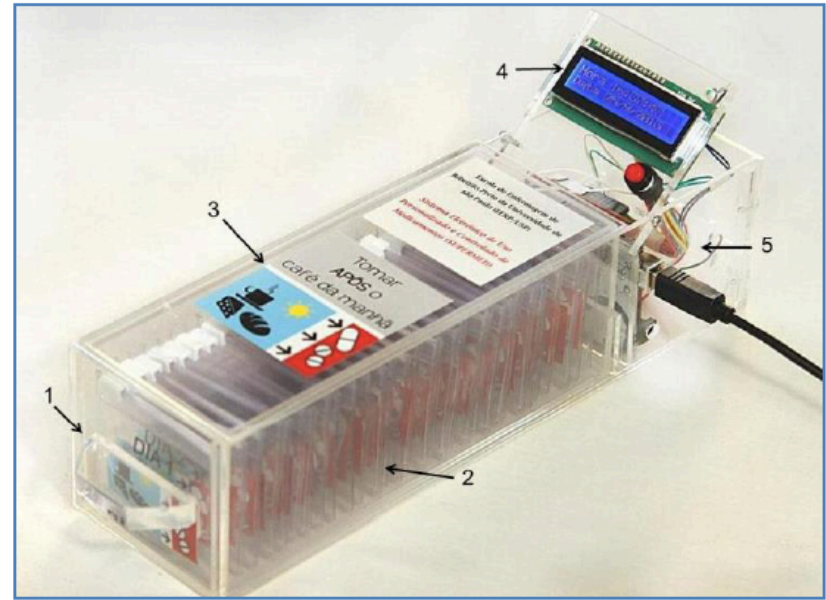

1- Caixa organizadora de medicamentos; 2- Sachês de medicamentos;

3- Etiquetas com informações para o uso correto; 4- Relógio com alarme;

5- Sistema eletrônico.

Fonte: VIEIRA (2013, p. 45)

Os medicamentos são separados e colocados em sachês (Figura 7) com identificação informando o dia, horário e dosagem. Horário e dosagem são representados com o uso de imagens (Figura 8).

Figura 7: Sachê de medicamentos.

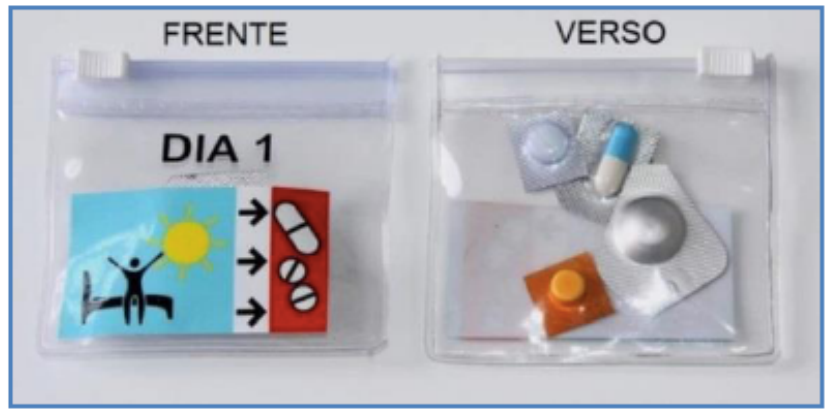

Fonte: VIEIRA (2013, p. 46) 
Figura 8: Etiquetas com informações corretas para uso dos medicamentos.
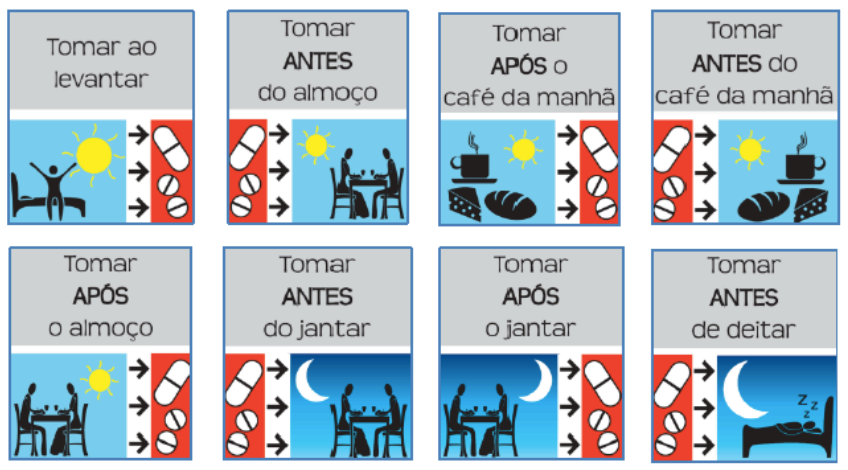

Fonte: $\operatorname{VIEIRA}(2013$, p. 46)

As figuras 9 e 10 apresentam o SUPERMED preparado para dois pacientes diferentes.

Figura 9: SUPERMED preparado para paciente com sete momentos distintos para tomar seus medicamentos.

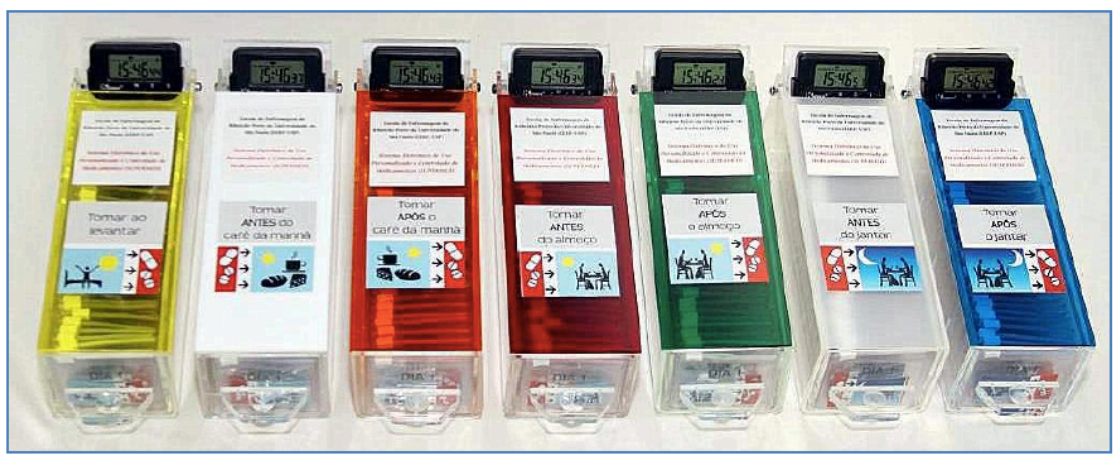

Fonte: VIEIRA (2013, p. 48)

Figura 10: SUPERMED preparado para paciente com três momentos distintos para tomar seus medicamentos.

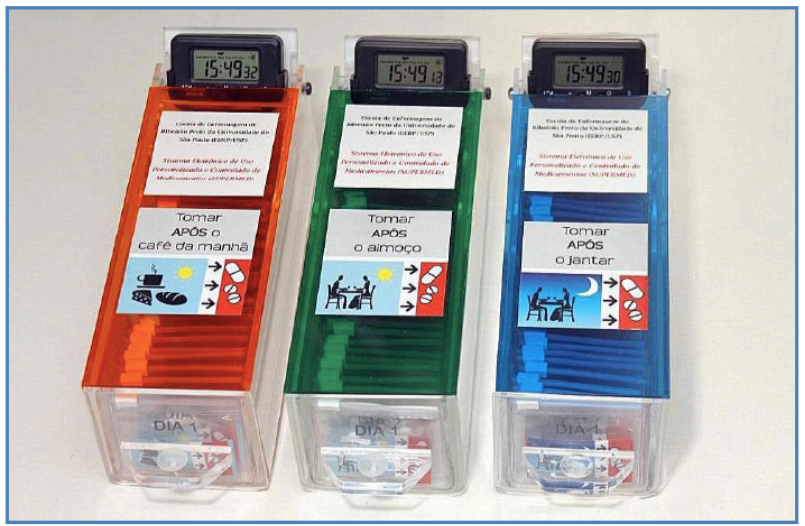

Fonte: VIEIRA (2013, p. 50) 


\subsection{Solução proposta por uma idosa - picotes em blister}

Como apresentado anteriormente, a estratégia para tomada dos medicamentos pode ser desenvolvida pelo próprio idoso onde ele mesmo personaliza as informações. Em um levantamento informal, realizado como parte de uma pesquisa de doutoramento, um relato chamou a atenção pela sua simplicidade e efetividade da personalização criada por uma idosa como estratégia para tomar os seus medicamentos. Ela relatou tomar apenas comprimidos, pela manhã, pela tarde e pela noite. No entanto, alguns comprimidos devem ser tomados uma vez ao dia, outros duas e alguns três. A estratégia adotada por ela foi realizar cortes no blister dos comprimidos a partir de uma codificação elaborada por ela, para identificar os comprimidos que devem ser tomados pela manhã (uma vez ao dia), de manhã e tarde (duas vezes ao dia) e manhã, tarde e noite (três vezes ao dia). A figura 11 ilustra a personalização elaborada pela idosa. A idosa não criou nenhum artefato novo de informação, mas fez intervenção no blister que ela já recebe com os medicamentos.

Figura 11: Corte no blister para identificar quanto e quando tomar o medicamento.

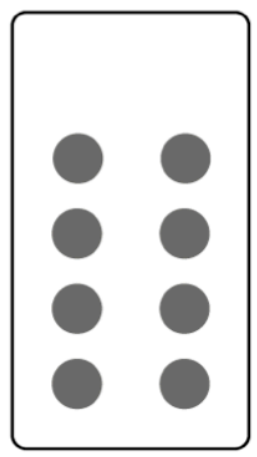

Blister original

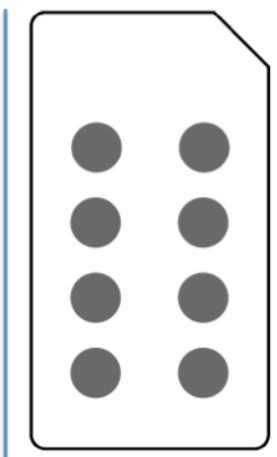

Manhã

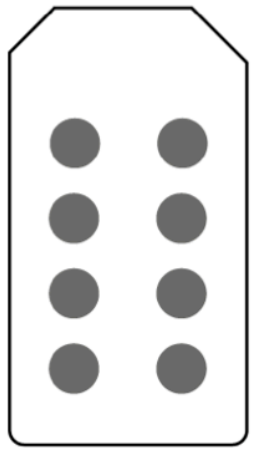

Manhã Tarde

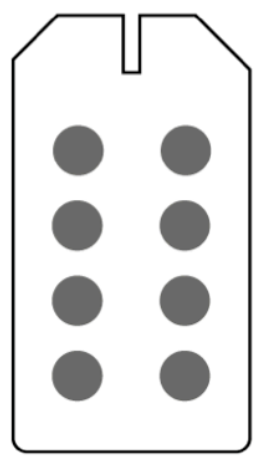

Manhã

Tarde e Noite

Fonte: Elaborado pelo autor (2018)

\section{Discussão}

Como foi apresentado anteriormente, os exemplos escolhidos sobre uso de medicamentos, particularmente para idosos polimedicados, objetivam melhorar a adesão ao tratamento. Considerando-se a complexidade do regime médico e duração do tratamento, enquanto fatores de adesão relacionados à terapia, os três exemplos apresentados procuram responder não apenas ao uso de medicamentos, mas ao uso de múltiplos medicamentos, por meio da organização e disponibilização de informações específicas para o idoso. Também respondem ao fator "esquecimento", relacionado ao paciente, bem comuns aos idosos. No entanto, podem não responder satisfatoriamente aos fatores "má compreensão das instruções de tratamento" e "conhecimento e habilidade inadequados em gerenciar o tratamento de doenças", uma vez que a compreensão é anterior ao uso dos medicamentos. Consequentemente, estas questões constituem uma lacuna no processo pois a não compreensão das instruções de tratamento podem levar a uma organização equivocada e uso incorreto dos medicamentos. 
No que diz respeito à personalização, os exemplos caracterizam-se como personalização de informação para uso de medicamentos por focarem especificamente nesta tarefa. A utilização de todas as soluções apresentadas nos exemplos está condicionada ao atendimento de cada idoso em particular, ou seja, de suas demandas, características, regime terapêutico etc. Desta forma, a utilização da solução depende de se personalizar (ou customizar) o seu sistema de informação para o idoso que irá utilizá-la. Se personalização puder ser considerada como "tornar pessoal", é possível observar uma certa gradação no processo ao se comparar as três soluções. Cada solução apresenta seu conjunto de elementos e a personalização é possível até o limite do sistema. O sistema de picote no blister, desenvolvido pela idosa, parece ser o "mais pessoal", não apenas por ser a criadora do sistema, mas pelo fato de estar mais próximo das características do paciente (fatores relacionados ao paciente). Neste artigo não se procurou fazer distinções entre os termos relacionados à ideia de personalização nem em se discutir em profundidade uma possível gradação no processo de personalização. Ambas as questões merecem tratamento específico e podem ser abordados em outro momento.

Por fim, pode-se perceber que as soluções estão ancoradas em informações visuais (e acrescidas de informações sonoras, no caso do SUPERMED), independente da formação e conhecimento de quem o elaborou. Cores, organização visual de elementos, tipografia, pictogramas, formas fazem parte intrínseca do sistema de informação resultante das soluções sem os quais seu uso seria inviabilizado.

\section{Considerações finais}

Após percorrer a literatura e analisar alguns exemplos de personalização de informação para idosos polimedicados, é possível dizer que os estudos sobre a temática abordada neste artigo são esparsos e pontuais, e que merecem ser melhor abordados. Considerando-se as dimensões da adesão apresentadas anteriormente, seria recomendável que os estudos pudessem ter uma abrangência sistêmica e aproximasse os diversos stakeholders envolvidos no processo de uso dos medicamentos.

Reforçando esta ideia, vários estudos que serviram de base para este artigo mencionam, muitas vezes de forma enfática, a importância de que a personalização e o processo de desenvolvimento dos artefatos sejam centrados no usuário idoso. A sua participação ativa no processo, desde a coleta de dados até a prototipação (em diversos níveis) e testes pode ser crucial a qualidade do uso de medicamentos pelo idoso polimedicado. A estratégia, a personalização e os artefatos resultantes poderão ser mais adequados para o idoso.

Como resultado, a personalização de informações aos idosos se mostra tão complexa quanto o universo em que vive e as demandas que apresenta em seu tratamento. Para que a aderência referente à dimensão relativa ao paciente possa ser melhorada no caso de idosos polimedicados, a personalização deve ser capaz de materializar uma estratégia de uso de medicamentos que seja eficaz e eficiente para o paciente, em especial quando é autônomo e faz a auto-gestão de seu tratamento. No entanto, o quanto personalizar é uma questão que deve ser melhor estudada e explorada.

Outra questão a ser considerada refere-se ao desenvolvimento de artefatos de informação em si mesmo. Como comentado anteriormente, não existe solução única e universal. Para cada idoso, uma estratégia, um ou mais artefatos específicos. Pode ser que, mais importante que 
desenvolver os artefatos seja refletir em como articular as inúmeras variáveis envolvidas para que a solução seja efetivamente personalizada.

Por fim, pode-se concluir que a personalização das informações sobre medicamentos, sobretudo para pacientes idosos, é um fator que pode contribuir significativamente para sua adesão aos medicamentos, mas que oferece ainda muitos desafios a serem enfrentados. Requer ainda muitos estudos e debates para que se encontre um equilíbrio entre a padronização e a personalização.

\section{Referências}

ADLER DESIGN. Clear Rx Medication System. In: Adler Design, 2018. (http://www.adlerdesign.com/project/clear-rx-medication-system/)

BERNARD, S. The Perfect Prescription. In: New York Best Doctors, 2018. (http://nymag.com/nymetro/health/features/11700/index1.html)

BOSWORTH, H. B. et al. Medication Adherence: A Call for Action. American Heart Journal, $n^{\circ} .162 .3$, p. 412-424, 2011.

CAVACO, A.; SANTOS, A. L. 2012. Avaliação da legibilidade de folhetos informativos e literacia em saúde. Rev. Saúde Pública, São Paulo, v. 46, nº. 5, out. 2012.

CAZARIM, M.S. ARAÚJO, A.L.A. O paciente idoso sob o aspecto da utilização de antimicrobianos: repercussão ao sistema público de saúde brasileiro (SUS). Rev Ciências Farmacêutica Básica Apl., 32(3), p. 305-311, 2011.

ENWALD, H. P. K.; NIEMELA, R.M.; KEINÄNEN-KIUKAANNIEMI, S.; LEPPÄLUOTO, J.; JÄMSÄ, T.; HERZIG, K. H.; OINAS-KUKKONEN, H.; HUOTARI M. A. A. Human information behaviour and physiological measurements. Health Information and Libraries Journal, $n^{\circ} .29, p p .131-140,2011$.

IBGE. Tábuas Completas de Mortalidade. In: IBGE - Instituto Brasileiro de Geografia e Estatística. 2014. (http://www.ibge.gov.br/home/estatistica/populacao/tabuadevida/2014/default.shtm).

GELLAD, W. F.; GRENARD, J. L., MARCUM, Z. A. A Systematic Review of Barriers to Medication Adherence in the Elderly: Looking Beyond Cost and Regimen Complexity. The American Journal of Geriatric Pharmacotherapy. Vol. 9, n.1, p. 11-23, 2011.

GUÉNETTE L; MOISAN J. Elderly people's knowledge of the purpose of their medicines. The American Journal of Geriatric Pharmacotherapy, $n^{\circ} .9(1)$, p. 49-57, 2011 Feb.

LIU, C.; KEMPER, S.; MCDOWD, J. The use of illustration to improve older adults' comprehension of health-related information: Is it helpful? Patient Education and Counseling, $n^{\circ}$. 76, p. 283-288, 2009.

MALONEY, K. W.; KAGAN, S. H. Adherence and Oral Agents with older patients. Seminars in Oncology Nursing, Vol 27, nº. 2 (May), p. 154-160, 2011.

MCLAUGHLIN, A. C.; MAYHORN, C. B. Designing effective risk communications for older adults. Safety Science, $n^{\circ} .61$, p. 59-65, 2014.

MINVIELLE E.; WAELLIB, M.; SICOTTEC, C.; KIMBERLY, J. R. Managing customization in health care: A framework derived from the services sector literature. Health Policy, $n^{\circ}$. 117, p. 216-227, 2014.

OLESEN, C., HARBIG, P., BARAT, I., DAMSGAARD, E.M. The impact of pharmaceutical care on non- 
adherence in elderly polypharmacy patients. In: 9th congress of the EUGMS / European Geriatric Medicine 4, 2013, p. S142-S216.

PFAFF, H.; DRILLER, E.; ERNSTMANN, N.; KARBACH, U.; KOWALSKI C.; SCHEIBLER, F.; OMMEN, O. Standardization and Individualization in Care for the Elderly: Proactive Behavior Through Individualized Standardization. Open Longevity Science, $n^{\circ}$. 4, p. 51-57, 2010.

PORTAL BRASIL. População idosa no Brasil cresce e diminui número de jovens, revela Censo. In: Governo do Brasil, 2013. (http://www.brasil.gov.br/noticias/arquivos/2011/04/29/populacaoidosa-no-brasil-cresce-e-diminui-numero-de-jovens-revela-censo)

SCHMITT JÚNIOR, A. A.; LINDNER, S.; SANTA HELENA, E. T. Avaliação da adesão terapêutica em idosos atendidos na atenção primária. Rev. Assoc. Med. Bras . vol. 59, nº 6, p. 614-621, 2013.

SIMÕES, C. M. O. Medicamentos em idosos. In: SCHENKEL, E. P.; MENGUE, S. S.; PETROVICK, P. R. Cuidados com os medicamentos. Florianópolis: Editora UFSC, 2012.

SNOWDON, A.; SCHNARR, K.; ALESSI, C. "It's All About Me": The Personalization of Health Systems. In: Ivey, 2014. (https://www.ivey.uwo.ca/cmsmedia/3467873/its-all-about-me-thepersonalization-of-health-systems.pdf).

SPATZ, M.A. Personalized Health Information: Patient-centered education includes the use of 'patient pathways' and tailored information. The American Journal of Nursing, Vol. 109, No. 4, p. 70-2, 2009.

UNNI, E. J.; FARRIS, K. B. Unintentional non-adherence and belief in medicines in older adults. Patient Education and Counseling, $n^{\circ} .83$, p. 265-268, 2011.

VIEIRA, L.B. Avaliação da adesão à terapêutica de pacientes idosos hipertensos antes e após o desenvolvimento e uso de um Sistema Eletrônico de Uso Personalizado e Controlado de Medicamentos. 2013. 125 f. Tese (Doutorado) - Escola de Enfermagem de Ribeirão Preto, USP, Ribeirão Preto, 2013

WAARDE, K. Visual information about medicines. Providing patients with relevant information. In: SPINILLO, C. G.; COUTINHO, S. (Org.). Selected Readings of the Information Design International Conference 2003. Recife: SBDI, p. 119 -120. 2004

WERLANG, M. C.; ARGIMON, I. I. L.; STEIN, L. M. Estratégias de Memória utilizadas por idosos para lembrarem do uso dos seus medicamentos. Estud. Interdiscip. Envelhec., Porto Alegre, v. 13, n. 1, p. 95-115, 2008.

WORLD HEALTH ORGANIZATION. Adherence to long-term therapies: evidence for action. Switzerland. 2003. 